\title{
Primary intraparenchymatous spinal cord melanoma
}

\author{
Jesús Vaquero, Félix de Prado and Manuel Pedrosa \\ Service of Neurosurgery, Puerta de Hierro Clinic, Autonomous University, Madrid, Spain
}

\begin{abstract}
A case of primary melanoma arising from the spinal cord parenchyma at the Th 10-11 level, in a 50-year-old female, is presented. In this patient, Magnetic Resonance Imaging (MRI) showed an intraparenchymatous lesion, with rounded borders, and an associated intramedullary cyst. On the basis of these findings and the long duration of symptoms, an erroneous preoperative diagnosis of intramedullary ependymoma was made.
\end{abstract}

Keywords: spinal cord; magnetic resonance imaging; spinal tumors; primary spinal cord melanoma

\section{Introduction}

Primary melanoma of the Central Nervous System is unusual. According to Rubino et $a l^{1}$ prior to 1993, less than 250 cases have been reported, including cases arising from leptomeninges, ${ }^{2-5}$ brain parenchyma, ${ }^{2-}$ $4,6-9$ choroid plexus, ${ }^{10}$ cerebellum or cerebellopontine angle, ${ }^{11-13}$ pituitary or suprasellar region, ${ }^{14,15}$ pineal area ${ }^{1,16}$ or spinal cord. ${ }^{2,6,17-21}$ The most important reviews on this subject clearly distinguish four different categories of primary melanoma affecting the Nervous System: (a) diffuse leptomeningeal melanomatosis, with infiltration of the leptomeninges of the brain and spinal cord; (b) discrete spinal cord melanomas; (c) primary intracranial isolated melanomas; and (d) melanosis of the meninges of the brain or spinal cord associated with cutaneous pigmentation (neurocutaneous melanosis). ${ }^{9}$

Among these categories, primary spinal melanoma represents a very rare clinicopathological entity. It was first reported by Hirschberg in $1906 .{ }^{18}$ In 1960 Hirano and Carton reviewed 25 patients in the literature ${ }^{17}$ and in 1972, Bergdahl et al, reporting 10 cases of primary malignant melanoma of the Central Nervous System, added three new cases involving the spinal cord. ${ }^{6}$ In 1987, Larson et al reported five new cases of primary spinal cord melanoma, and reviewed another 26 cases from the literature ${ }^{20}$ and in 1989, Yamasaki et al reported a new case. ${ }^{21}$ Since then, according to a literature search, new cases of primary spinal cord melanoma have been not reported. Furthermore, Magnetic resonance imaging (MRI) characteristics of primary spinal cord melanoma have been not assessed. For this reason, we present a new case of primary spinal cord melanoma which has been studied by means of MRI. In this patient, an erroneous preoperative diagnosis of ependymoma was made.

Correspondence: Dr J Vaquero

\section{Case report}

This 50 year-old female patient was referred to us for surgical treatment of what appeared to be a spinal cord ependymoma. Her symptoms had started 2 years earlier with progressive paresis of the right leg. When admitted, neurological examination revealed paresis of the right leg, with hyper-reflexia and a Babinski sign. On the left side, hypoesthesia was recorded from Th10 level.

Magnetic resonance imaging showed an intraparenchymatous intramedullary tumor, with rounded borders, at the Th10-Th11 level. On the T1-weighted image the lesion showed homogeneous high intensity which was enhanced after gadolinium diethylenetriamine penta-acetic acid contrast administration. On the T2-weighted image, the lesion was hypointense, showing a central area of hyperintensity. A cyst was observed on the upper border of the tumor (Figure 1). With these data, a presumptive diagnosis of intramedullary ependymoma was established. A laminectomy was performed and after myelotomy, a black totally intramedullary tumor was disclosed. The tumor had necrotic areas and had poorly defined margins. Pathological study of the subtotally removed tumor showed a typical melanoma (Figure 2). After extensive studies including cerebral MRI and abdominal CTscan, other foci of melanoma were not found. With these data, a diagnosis of primary spinal cord melanoma was established, and radiation therapy to the whole brain and the entire neuroaxis was done. After operation, the patient experienced weakness of both legs, with only limited voluntary flexion and extension of her right foot; the hypoesthesia had increased to total anesthesia, with a sensory level at Th10, and permanent urinary catheterization was necessary. Subsequently she began to recover flexion of right leg, but with no changes in her sensory level. She was referred to a Rehabilitation Center. Now, 15 months after surgery, there has been no substantial improvement in the neurological deficits. At present, the patient is capable of a certain degree of voluntary 


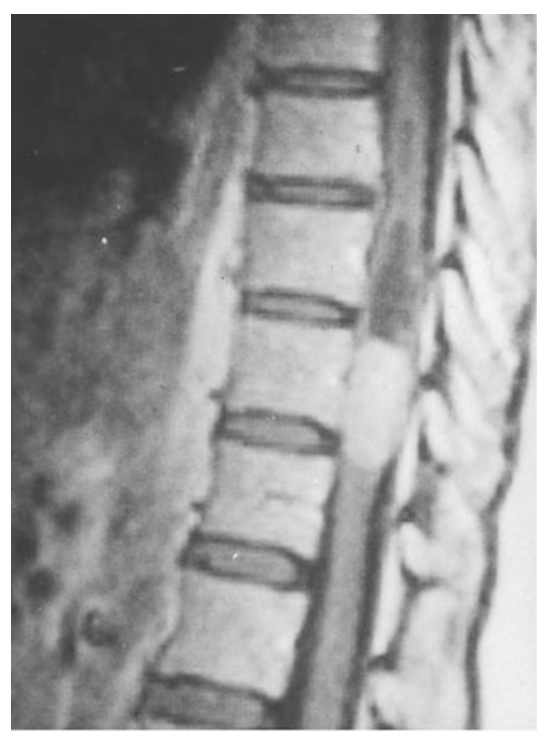

b

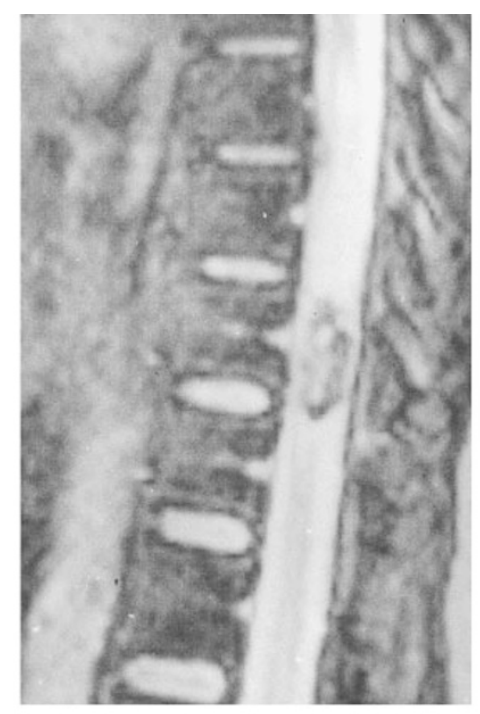

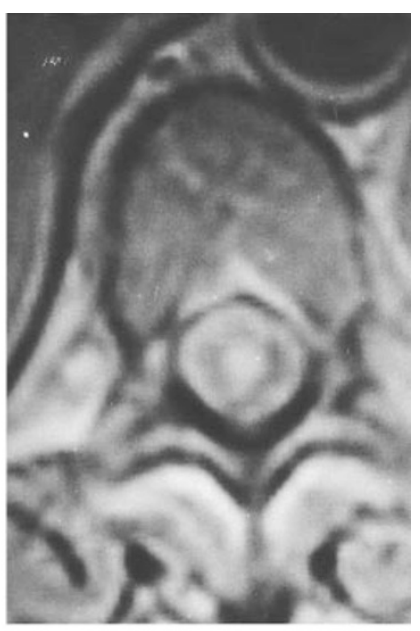

Figure 1 Magnetic resonance images of the intraparenchymatous intramedullary tumor at Th10-Th11 level. Left: Sagittal T1weighted image, after gadolinium diethylenetriamine penta-acetic acid administration, showing the rounded margins of the tumor with an associated cyst. Center: Sagittal T2-weighted image showing the hypointensity of the tumor, with a central area of hyperintensity. Right: Axial T1-weighted image, after contrast administration, showing the central intramedullary localization of the tumor

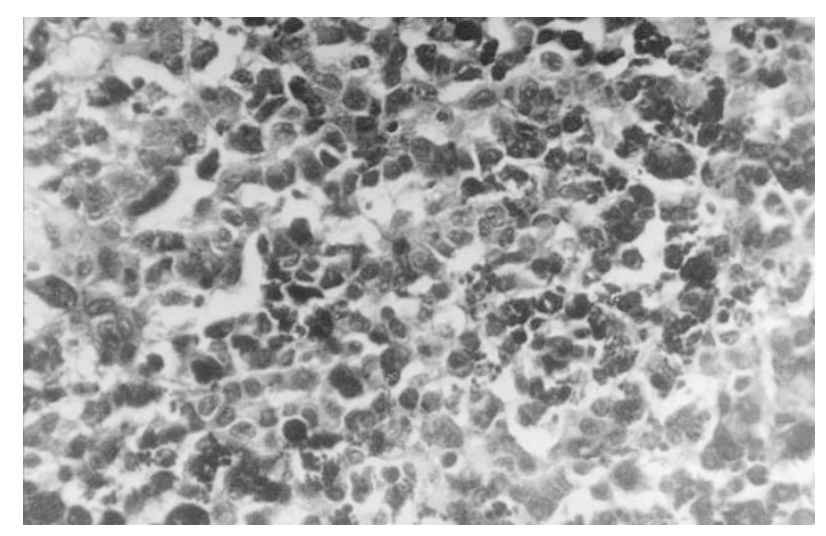

Figure 2 Microscopical aspect of the resected tumor, showing a malignant melanoma composed of tumor cells with melanin pigmentation and polymorphism. H.E. technique, $\times 200$

flexion and extension of her left leg, but she is unable to move her right leg. She is unable to remain standing and requires a wheelchair for mobility, and requires permanent urinary catheterization. She continues with a sensory level at Th10, hyperreflexia of both legs and a Babinski sign. At this time, there is no evidence of any neoplasia.

\section{Discussion}

According to the criteria established by Hayward ${ }^{3}$ (absence of malignant melanoma, metastatic or primary, outside the central nervous system, and pathological confirmation of the tumor) our present case can be considered as a primary melanoma arising from the nervous tissue of the spinal cord.

Most primary nervous system melanomas are either obviously meningeal in location or are so superficially situated within the parenchyma that a meningeal origin is assumed, arising from the pial melanocytes. ${ }^{2,4}$ According to this theory, it may be assumed that intraparenchymatous primary melanomas originate from melanoblasts accompanying the pial sheaths of vascular bundles. ${ }^{3}$ Another theory proposes that, during embryogenesis, a few neuroectodermal rest cells migrate to reside within the neural tube and its coverings. ${ }^{8}$ In this way, melanoblasts can reach the nervous parenchyma and may be the origin of the rare cases of primary melanoma of the brain or spinal cord. In our case, a relation to meninges was not found and this fact suggests that the tumor originated from perivascular melanoblasts or from free melanoblasts within the nervous tissue.

The clinical features in our case agree with those of others who have noted that primary melanomas of the spinal cord occur most frequently in the middle and lower thoracic cord, causing an asymmetrical myelopathy. ${ }^{20}$ On the other hand, it has been reported that primary spinal melanomas present a very different clinical course than those of the more common melanoma of the skin with metastases to the central nervous system, which is usually fatal within 6 months because of widespread metastases. In our case, the long duration of symptoms supports the opinion that 
primary spinal melanoma presents an indolent course. Moreover, primary melanomas of the spinal cord seem to be associated with a longer survival than do those primarily situated in the brain, and may be associated with several years of survival after diagnosis. ${ }^{6,20}$

The magnetic resonance characteristics of intracranial melanomas have been discussed by Uematsu et al, who reported a case of meningeal melanocytoma, showing homogeneous high intensity on the T1weighted image and low intensity on the T2-weighted image. 5 Paramagnetic stable free radicals, such as indole semiquinones and semiquinonimines are present in melanocytic tumors causing a decrease in the T1 and T2 relaxation times. Nevertheless, previous areas of hemorrhage may have a greater influence on the magnetic resonance appearance than does melanin, resulting in heterogeneous signal intensity. Therefore, it is obvious that the magnetic resonance appearance of melanoma is not uniform, but depends on the degree of previous hemorrhage and melanization within the tumor. In our patient, the signal pattern on MRI was opposite to that usually seen with intraparenchymatous nervous system tumors, which appear isointense or hypointense on the T1-weighted image and hyperintense on the T2-weighted image. Nevertheless, the long duration of symptoms, the rounded borders of the tumor and the presence of an associated intramedullary cyst suggested an ependymoma with chronic intratumoral hemorrhage. The main conclusion that we have obtained with our present observation is that, independently of the long duration of symptoms, the possibility of a primary malignant melanoma must be considered when MRI study discloses an intraparenchymatous spinal cord tumor with apparently rounded and well delimited margins.

\section{References}

1 Rubino GJ et al. Primary pineal melanoma: Case report. Neurosurgery 1993; 33: $511-515$.
2 Burger PC, Scheithauer BW. Atlas of Tumor Pathology. Tumors of the Central Nervous System. Armed Forces Insitute of Pathology, Washington DC, 1994.

3 Hayward RD. Malignant melanoma and the central nervous system. A guide for classification based on the clinical findings. $J$ Neurol Neurosurg Psychiatry 1976; 39: 526-530.

4 Russell DS, Rubinstein LJ. Pathology of Tumours of the Nervous System. Edward Arnold, London. 1989.

5 Uematsu Y et al. Meningeal melanocytoma: magnetic resonance imaging characteristics and pathological features. Case report. $J$ Neurosurg 1992; 76: 705-709.

6 Bergdhal L et al. Primary malignant melanoma of the central nervous system. A report of 10 cases, Acta Neurochir (Wien) 1972; 26: $139-149$.

7 Nakagawa $\mathrm{H}$ et al. Long-term survival after removal of primary intracranial malignant melanoma. Case report. Acta Neurochir (Wien) 1989; 101: 84-88.

8 Pappenheim E, Bhattacharji SK. Primary melanoma of the central nervous system. Arch Neurol 1962; 7: 101-113.

9 Rodríguez Baena $\mathrm{R}$ et al. Primary solitary intracranial melanoma: Case report and review of the literature. Surg Neurol 1992; 38: $26-37$.

10 Beatty RA. Malignant melanoma of the choroid plexus epithelium. J Neurosurg 1972; 36: $344-347$.

11 Pasquier B et al. Primary malignant melanoma of the cerebellum. Cancer 1978; 41: 344-351.

12 Salm R. Primary malignant melanoma of the cerebellum. $J$ Pathol Bacteriol 1967; 94: 196 - 200.

13 Vasdev A et al. Apparently primary malignant melanoma of the cerebellopontine angle. J Neuroradiol 1990; 17: $152-156$.

14 Copeland DD, Sink JD, Seigler HF. Primary intracranial melanoma presenting as a suprasellar tumor. Neurosurgery 1980; 6: $542-545$.

15 Scholtz CL, Siu K. Melanoma of the pituitary. J Neurosurg 1976; 45: $101-103$.

16 Weindling SM, Press GA, Hesselink JR. MR characteristics of a primary melanoma of the quadrigeminal plate. AJNR 1988; 9: $214-215$.

17 Hirano A, Carton CA. Primary malignant melanoma of the spinal cord. J Neurosurg 1960; 17: $935-944$.

18 Hirschberg A. Chromatophoroma medullae spinalis. Ein Beitrag zur Kenntnis der primãren Chromatophorome des Zentralnervensystems. Virchows Arch 1906; 186: 229-240.

19 Kiel FW, Starr LB, Hansen JL. Primary melanoma of the spinal cord. J Neurosurg 1961; 18: 616-629.

20 Larson TC, Houser W, Onofrio BM, Piepgras DG. Primary spinal melanoma. J Neurosurg 1987; 6: 47-49.

21 Yamasaki $\mathrm{T}$ et al. Primary spinal intramedullary malignant melanoma: Case report. Neurosurgery 1989; 25: 117-121. 